\title{
MESH CONVERGENCE STUDY FOR 2-D STRAIGHT-BLADE VERTICAL AXIS WIND TURBINE SIMULATIONS AND ESTIMATION FOR 3-D SIMULATIONS
}

\author{
Saman Naghib Zadeh, Matin Komeili and Marius Paraschivoiu \\ Mechanical and Industrial Engineering, Concordia University, Montreal, Canada \\ E-mail: marius.paraschivoiu@concordia.ca
}

Received October 2013, Accepted September 2014

No. 13-CSME-167, E.I.C. Accession E.I.C. 3625

\begin{abstract}
Mesh resolution requirements are investigated for 2-D and 3-D simulations of the complex flow around a straight-blade vertical axis wind turbine (VAWT). The resulting flow, which may include large separation flows over the blades, dynamic stall, and wake-blade interaction, is simulated by an Unsteady ReynoldsAveraged Navier-Stokes analysis, based on the Spalart-Allmaras (S-A) turbulence model. A grid resolution study is conducted on 2-D grids to examine the convergence of the CFD model. Hence, an averagedgrid residual of $y^{+}>30$ is employed, along with a wall treatment, to capture the near-wall region's flow structures. Furthermore a 3-D simulation on a coarse grid of the VAWT model is performed in order to explore the influence of the 3-D effects on the aerodynamic performance of the turbine. Finally, based on the 2-D grid convergence study and the 3-D results, the required computational time and mesh to simulate 3-D VAWT accurately is proposed.
\end{abstract}

Keywords: vertical axis wind turbine; aerodynamic; grid convergence; tip vortex.

\section{ÉTUDE DE LA CONVERGENCE DU MAILLAGE 2D ET RÉSOLUTION SOMMAIRE 3D DE L'ÉCOULEMENT AUTOUR D'UNE ÉOLIENNE À AXE VERTICAL À PALES DROITES}

\section{RÉSUMÉ}

Les exigences en matière de convergence du maillage sont étudiées pour les simulations 2D et 3D de l'écoulement complexe autour d'une éolienne à axe vertical à pales droites (VAWT). Cet écoulement peut inclure une région de séparation sur les pales, du décrochage dynamique ainsi que de l'interaction pale sillage. Cet écoulement est modélisé par l'équation instationnaire de Navier-Stokes - moyennées à la Reynolds et utilise le modèle de turbulence Spalart-Allmaras (S-A). Une étude de la convergence du maillage est menée sur un maillage 2D pour examiner la convergence spatiale du modèle mécanique des fluides numérique (MFN) utilisé. Afin de modéliser l'écoulement près de la paroi, un maillage avec $y^{+}>30$ est employé avec un traitement de loi-log. De plus, une simulation sur un maillage grossière 3D d'une turbine VAWT est réalisée afin d'étudier l'effet 3D sur la performance aérodynamique de la turbine. Par la suite, basé sur l'étude de convergence du maillage en 2D et les résultats en 3D, le temps de calcul requis et le maillage nécessaire est conseillé afin d'obtenir une solution précise en 3D de l'écoulement autour d'une turbine.

Mots-clés : éolienne à axe vertical; aérodynamique; convergence du maillage; vortex bout d'aile. 


\section{INTRODUCTION}

Computational Fluid Dynamic (CFD) tools have proved to be very valuable for analysis and design of fluid dynamic systems for the last two decades. This popularity has benefited from many developments in High Performance Computing (HPC), both on the algorithm part as well as the hardware part. The accuracy of the simulation depends on the spatial discretization and the order of the discretization method used. For any given method and simulation problem it is essential to use a mesh that is fine enough so that the error is small but also to have the error in the asymptotic regime, meaning that it decreases with a given order as the mesh increases. In many industrial applications the mesh required to be in this regime is not trivial.

Furthermore, the mesh resolution requirement is more confusing for some turbulence models such as Large Eddy Simulation (LES) or Direct Numerical Simulation (DNS). It also plays an undeniable role in the accuracy of RANS turbulence methods. The grid should be fine enough to capture all the Subgrid-scale turbulence (DNS) or a portion of them (LES); in other words, the smallest grid has to be almost of the same order of magnitude as the Kolmogorov length scale. Therefore, it is too expensive to use these methods for complex geometry such as VAWT, where they work in moderate and high Reynolds number. In RANS models mesh should be small enough to capture the turbulence eddies in the large scale only. In fact, in the URANS model, large eddies are resolved and the turbulence model is used to consider the Subgrid-scale eddies' influence on the flow. That makes URANS methods an affordable and appropriate choice for most engineering applications such as airplane or wind turbine simulations.

The simulation of VAWT is one of the most challenging aerodynamic problems in the incompressible flow regime. Continuous variation of angle of attack results in rapid changes of the pressure distribution on the blade, and often dynamic stall occurs for low ratio speeds. In addition, vortices shedding from the blade on the windward side of the wind turbine travel downstream and they may interact with the blades on the leeward side. The flow gets even more complicated for 3-D simulations where tip vortices and other 3-D vortices should be taken into account correctly. Many of aforementioned influential aerodynamic phenomena are related to the turbulence features of the flow. Therefore, choosing an accurate turbulence simulation of flow is a key factor for prediction of the aerodynamic forces on the blades. However, any turbulence model requires sufficient mesh resolution, which will be explored here.

Most CFD simulations of flow around VAWT have been carried out in the last two decades and show significant discrepancies. McLaren et al. [1] used a commercial CFX solver to estimate the dynamic loading of the straight blades of a small-sized VAWT. They utilized the SST $k-\omega$ model in combination with the $\gamma-\theta$ transition model introduced by Menter et al. [2], in order to calculate lift and drag forces on the blades, and they declared that the transitional method captured more features of the flow. Ajedegba [3] investigated the power coefficient of a special type of small vertical axis wind turbine (Zephyr) at different tip speed ratios. He used the commercial software FLUENT with multiple reference frames (MRF). The simulations were carried out on a 2-D domain using the $k-\varepsilon$ model along with standard wall function. Although there was a relatively good agreement between CFD results and the stream tube model at low tip speed ratio range, the deviation from experiments is observed for higher tip speed ratios. The weakness of the $k-\varepsilon$ model in simulating intense adverse pressure gradient flows and complex geometries are the main reasons behind the discrepancy with experimental data.

Camelli and Lohner [4] introduced a new computation model that combined Baldwin-Lomax with Smagorinsky (BLS) to capture the separation on a circle in 2-D and a cylinder in 3-D. They also analysed the turbulence structures inside the wake behind the obstacles. Their model utilized Baldwin-Lomax in the near wall region and the Smogorinsky model for the far-wall region. They concluded that both Balwin-Lomax and BLS produce similar results and that their predictions are closer to the experimental data compared to when the Smagorinsky model is applied alone, due to coarse grid in the near-wall region. Jiang et al. [5]

used BLS to investigate the effects of the number of blades and tip speed ratio on the power coefficient of 
a small-sized straight Darrieus wind turbine. Although their results demonstrated, qualitatively, the effect of solidity and the number of blades on the power coefficient of the turbine, they could not capture the stall on the blades. The drawback correlates to the fact that BLS has the same deficiencies as the BL model in the solution of flow, including the large separation region and significant curvature effects. Ferreira et al. [6] simulated the air flow through the VAWT turbine with different turbulence models and validated the results with experimental observations obtained with the particle image velocimetry (PIV) technique. They investigated, specifically, the part of rotation in which dynamic stall happens. Out of the CFD models that they used (RANS with Spalart-Allmaras, RANS with $k-\varepsilon$, LES with Lilly SGS model, DES with S-A near-wall region), DES resulted in the best agreement with the experiment. Although using LES or DES simulation with sufficient grid resolution may result in more accurate flow prediction compared to URANS models, a significant obstacle to using these methods, in practical for wind turbine problems, is the availability of computer resources. The problem gets more severe for simulations aimed at capturing the 3-D turbulence features such as tip vortices. Consequently, the only affordable turbulence model for 3-D VAWT simulation is tied to URANS models. It is clear, from all these works, that the mesh convergence is not always achieved. Therefore it is important to provide some clarification related to the meshes required from accurate VAWT simulations.

Herein, 2-D and 3-D meshes are studied for the Spalart-Allmaras turbulence model. In the grid studies the power coefficient extracted from VAWT is chosen as the objective variable. Therefore the main changes on the grid are concentrated in the near-rotor region. For the 2-D simulations the torque generated by each blade and the total power coefficient are calculated. Then, a grid convergence is conducted, based on the power coefficient, to estimate the sensitivity of the model to the grid resolution. A 3-D simulation is also accomplished to show the 3-D turbulence influences on VAWT performance.

Grid study is an inevitable step of a CFD simulation. Grid Convergence Index (GCI) is a popular grid study method among engineers to estimate the grid convergence of a discretization method. GCI was first introduced by Roache [7] and it has been used in many papers since to study the grid residual. Paciorri et al. [8] used GCI to calculate the desirable residual for a junction flow. Later, other scientists also developed the GCI technique. Biron et al. [9] compared three types of mesh by using a GCI method suggested by Hardy et al. [10].

In the following sections first the governing equations and the numerical methodology are presented. Then, the numerical domain, boundary conditions and grid properties are shown. After that the convergence study, based on 2-D results, is conducted. A 3-D simulation on a coarse mesh focuses on capturing the tip vortices. Finally, the desirable 3-D grid size is proposed to simulate a VAWT using the S-A method as a turbulence model.

\section{GOVERNING EQUATIONS}

The simulation of flow over VAWT is studied by solving the Unsteady Reynolds-Averaged Navier-Stokes equations (URANS) with S-A as the turbulence model. Applying Reynolds decomposition and taking time-average of the continuity and momentum equations yields the following URANS equations for incompressible flows

$$
\begin{gathered}
\frac{\partial \bar{u}_{i}}{\partial x_{i}}=0 \\
\frac{\partial \bar{u}_{i}}{\partial t}+\bar{u}_{j} \frac{\partial \bar{u}_{i}}{\partial x_{j}}=-\frac{1}{\rho} \frac{\partial \bar{P}}{\partial x_{i}}+v \frac{\partial^{2} \bar{u}_{i}}{\partial x_{j} \partial x_{j}}+\frac{\partial \tau_{i j}}{\partial x_{j}}
\end{gathered}
$$


where $\bar{P}$ and $\bar{U}_{i}$ are the average pressure and velocity components, respectively, $v$ is the kinematic viscosity and $\rho$ is the fluid density. Furthermore, $\tau_{i j}$ is the specific Reynolds Stress tensor and can be described as

$$
\tau_{i j}=-\overline{u_{i}^{\prime} u_{j}^{\prime}} \text {. }
$$

This symmetric tensor has six independent components and expresses the correlation between the fluctuating velocities. Basically, for 3-D flows, there are four equations and ten unknowns including six components for Reynolds stress, one pressure and three velocities. Therefore, in order to close the system, more equations are needed.

\subsection{Turbulence Modelling Methodology}

The next approach is to employ the Spalart-Allmaras turbulence model [11] as an additional transport equation. In this model the turbulent kinematic eddy viscosity term is expressed by the following equation:

$$
v_{t}=\tilde{v} f_{v 1}
$$

where $\tilde{v}$ and $f_{v 1}$ are modified kinematic eddy viscosity and closure function, respectively and can be expressed by

$$
\begin{gathered}
f_{v 1}=\frac{\chi^{3}}{\chi^{3}+C_{v 1}^{3}}, \\
\chi=\frac{\tilde{v}}{v} .
\end{gathered}
$$

Here, $v$ is kinematic molecular viscosity and $C_{v 1}$ is a constant. The transport eddy viscosity equation in the $\mathrm{S}-\mathrm{A}$ model can be defined by the following formula:

$$
\frac{\partial \tilde{v}}{\partial t}+U_{j} \frac{\partial \tilde{v}}{\partial x_{j}}=C_{b 1} \tilde{s} \tilde{v}-C_{w 1} f_{w}\left(\frac{\tilde{v}}{d}\right)^{2}+\frac{1}{\sigma} \frac{\partial}{\partial x_{k}}\left[(v+\tilde{v}) \frac{\partial \tilde{v}}{\partial x_{k}}\right]+\frac{C_{b 2}}{\sigma} \frac{\partial \tilde{v}}{\partial x_{k}} \frac{\partial \tilde{v}}{\partial x_{k}}
$$

In this equation, the first three terms on the right-hand side are the production, destruction and diffusion of the kinematic eddy viscosity, respectively. The diffusion term includes both the molecular viscosity and the turbulent structures effects. The production term $\tilde{s}$ is

$$
\tilde{s}=f_{v 3} \Omega+\frac{\tilde{v}}{k^{2} d^{2}} f_{v 2}
$$

where $\Omega$ corresponds to the magnitude of vorticity and $d$ is the point field distance to the nearest wall, and

$$
\begin{gathered}
f_{v 2}=\frac{1}{\left(1+\chi / c_{v 2}\right)^{3}}, \\
f_{v 3}=\frac{\left(1+\chi f_{v 1}\right)\left(1-f_{v 2}\right)}{\chi} .
\end{gathered}
$$

The destruction function can be defined as,

$$
f_{w}=g\left(\frac{1+C_{w 3}^{6}}{g^{6}+C_{w 3}^{6}}\right)^{1 / 6}
$$

where,

$$
\begin{gathered}
g=r+C_{w 2}\left(r^{6}-r\right), \\
r=\min \left[\frac{\tilde{v}}{\tilde{s} k^{2} d^{2}}, 10\right] .
\end{gathered}
$$

The constants corresponding to the S-A model are listed in Table 1. 
Table 1. S-A model coefficient.

\begin{tabular}{cc}
\hline$C_{b 1}=0.1355$ & $C_{v 1}=7.1$ \\
$C_{b 2}=0.622$ & $C_{v 2}=5.0$ \\
$C_{w 1}=\frac{C_{b l}}{k^{2}}+\left(1+C_{b 2}\right) / \sigma$ \\
$C_{w 2}=0.3$ & $k=0.41$ \\
$C_{w 3}=2.0$ & $\sigma=2 / 3$ \\
\hline
\end{tabular}

\subsection{Wall Treatment}

In order to capture the near-wall region's flow structures, the standard wall function for the S-A model is employed as follows:

$$
v_{t}=v\left(\frac{y^{+} \kappa}{\ln E y^{+}}-1\right)
$$

where $\kappa=0.4187$ and $E=9$ is the additive constant utilized in the logarithmic law of the wall. Using the wall function allows us to locate the first point adjacent to the wall in the logarithmic zone instead of the sub-layer. Therefore, the averaged $y^{+}$calculated should be around 30 in all simulations.

\section{NUMERICAL IMPLEMENTATION}

An OpenFoam ${ }^{\circledR}$ Solver [12] is used as our CFD tool in order to simulate the flow around the wind turbine. In the following sections the details of the finite volume technique that is used to discretize the governing equation is described and the linear solver is explained.

\subsection{Discretization Schemes}

In order to discretize convection terms in the velocity and turbulence equations, a Gauss scheme with upwind interpolation is used. Furthermore, an Euler implicit scheme is employed for the time discretization. The pressure gradient is discretized by using Gaussian integration followed by the linear interpolation scheme wherein second-order central differencing is used. Viscous terms are discretized by Gaussian integration with linear interpolation of the diffusion coefficient with a surface normal gradient scheme, which is an explicit non-orthogonal correction. It should be mentioned that the gradient normal to a surface is computed at cell faces. The approach is based on interpolating the cell gradients at the face intersection. The SIMPLE algorithm is mostly used for transient-state simulations to couple the velocity and pressure in OpenFOAM@ software.

\subsection{Numerical Solvers}

In order to solve the linear system of equations, the Krylov Subspace Solvers (KSS) are employed. The Preconditioned Bi-Conjugate Gradient (PBiCG) approach followed by the diagonal-based incomplete lowerupper (LU) preconditioner for asymmetric matrices is used for velocity and turbulence equations. For the pressure a Preconditioned Conjugate Gradient (PCG) solver with a diagonal-based incomplete Cholesky preconditioner is employed. These settings are described in [12].

\section{COMPUTATIONAL DOMAIN, GRID GENERATION AND BOUNDARY CONDITION}

In the present work, unsteady flow simulations are performed for 2-D and 3-D computational domains of a VAWT with the geometry specifications given in Table 2. Gambit software is used as a tool for creating the geometry and mesh generation. 
Table 2. Specification of the geometry.

\begin{tabular}{lc} 
Number of rotors & 1 \\
Number of blades & 4 \\
Blade chord & $0.4445 \mathrm{~m}$ \\
Rotor Diameter (D) & $5.395 \mathrm{~m}$ \\
Angular velocity of the rotor & $90 \mathrm{rpm}$ \\
Diameter of the rotating zone & $1.053 \mathrm{D}$ \\
\hline
\end{tabular}

Table 3. Far-field air flow properties.

\begin{tabular}{ll}
\hline Properties & Value \\
\hline Kinematic viscosity $(v)$ & $1.57 \times 10^{-5}\left(\mathrm{~m}^{2} / \mathrm{s}\right)$ \\
Density $(\rho)$ & $1.1774\left(\mathrm{~kg} / \mathrm{m}^{3}\right)$ \\
\hline
\end{tabular}

The computational domain of a VAWT includes two main zones, namely, a stationary zone, which is considered for the far field flow, and a rotating zone that rotates with the given angular velocity. A rectangular domain, which corresponds to the stationary zone, is utilized for the 2-D grid, with a distance of 10 rotor diameters from the axis of rotation to the top, bottom and left boundaries (Fig. 1a). There is a 15 rotor diameter distance from the axis of rotation to the boundary on the right. Figures $1 \mathrm{a}$ and $1 \mathrm{~b}$ illustrate the mesh of the computational domain as well as the mesh around an airfoil, respectively. The Bottom-Up approach is used to make the 3-D grid. In this technique the vertices, edges, and faces are first created in 2-D and thereafter 3-D meshes and volumes are constructed. We extend the 2-D domain by 1.71 chords in a span-wise direction and after that the grid is extended another 1.71 chords from the tip of the blade to the front in the $z$-direction (Fig. 1c).

In order to conduct CFD analysis, it is necessary to specify initial and boundary conditions for the domain. A fixed value is set for velocity at the far-field boundary upstream of the rotor and atmospheric pressure is specified for the pressure at the downstream boundary. Other air properties at the far field are listed in Table 3. A no-slip boundary condition is assumed on the blades; and at the top and bottom of the domain, a symmetry condition is employed. In the case of the 3-D domain the symmetry boundary is chosen for the back face, where the blades are attached, and slip boundary conditions are chosen at the opposite face. The modified kinematic eddy viscosity $(\tilde{v})$ is chosen as $\tilde{v}=5 v$ at the inlet and $v=0$ at the walls. Similarly, turbulent viscosity $\left(v_{t}\right)$ is computed from Equation (4) and set to be $v_{t}=2.029 e-05 \mathrm{~m}^{2} / \mathrm{s}$ at the inlet.

In the present study, General Grid Interface (GGI), developed by Beaudoin and Jasak [13], is used to couple the stationary and rotating zones. The methodology is based on weighted interpolation to compute and transmit the flow variables in the interface region.

\section{VERTICAL AXIS WIND TURBINE SIMULATION}

In this section first the grid convergence methodology is described and then it is implemented for 2-D simulations. Afterward, comparisons and differences between 2-D and 3-D simulations are studied.

\subsection{2-D Simulation}

In this section, the intent is to define the key parameters in wind turbine analysis. One of the most important parameters in wind turbine investigations is the Tip Speed Ratio (TSR), which is defined as the ratio of tip speed of the blade to the wind speed,

$$
\lambda=\frac{\omega R}{V}
$$




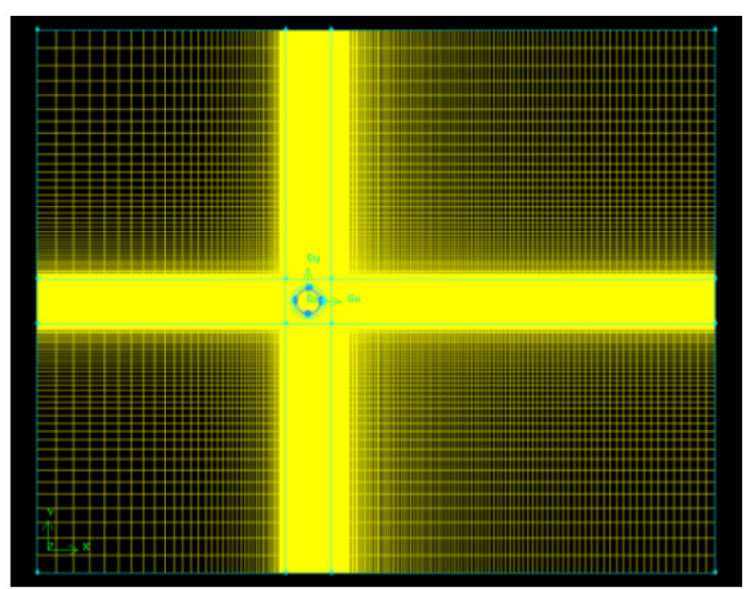

(a)

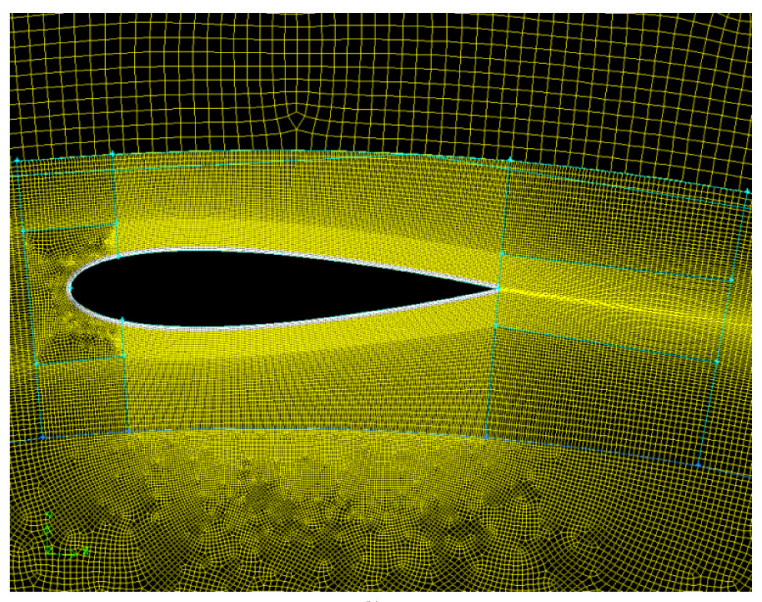

(b)

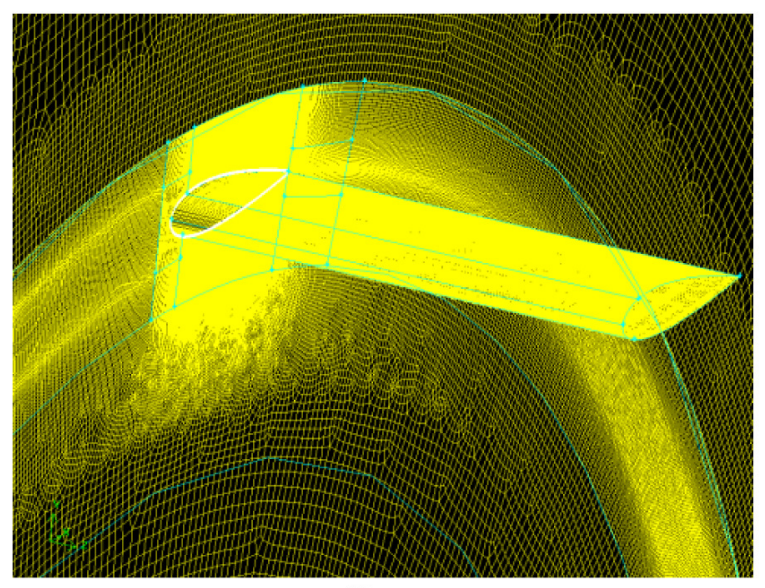

(c)

Fig. 1. Mesh; (a) computational domain; (b) blade; (c) span.

where $R$ is the rotor radius, $\omega$ is angular velocity of turbine, and $V$ is wind velocity.

Power coefficient $\left(C_{p}\right)$ is the quantity of interest, which can be derived by applying the Pi-Buckingham theorem, and is given by

$$
C_{p}=\frac{P}{\frac{1}{2} \rho A V^{3}}
$$

Here, $P$ is power, $\rho$ is the wind density, $A$ is the frontal swept area, $V$ and is the free stream velocity. In order to calculate the coefficient of power for various tip speed ratios, a fixed angular velocity of $90 \mathrm{rpm}$ as well as different wind velocities are employed. The torque is calculated by means of the developed code in OpenFOAM®, defined as the sum of the forces acting on each blade. In order to compute the coefficient of power a one-meter span is assumed for the swept area calculation.

The vorticity contours, at different blade locations, are depicted in Fig. 2 for TSR $=2$ and TSR $=3$. For a fixed angular velocity the average time step is $\approx 0.0003 \mathrm{~s}$, therefore at each time step the blades rotate $\approx 0.67^{\circ}$. In Fig. $2 \mathrm{a}$, the upwind blade is perpendicular to the flow direction. Figure $2 \mathrm{~b}$ shows the flow after a $30^{\circ}$ rotation from the previous position and Fig. $2 \mathrm{c}$ shows the flow after another $30^{\circ}$ rotation of the blades. It is observed that clockwise vortices at the top and counter-clock-wise vortices at the bottom are generated and travel downstream by the mainstream flow and gradually dissipate. Figure 2 also shows the 
$\mathrm{TSR}=2$
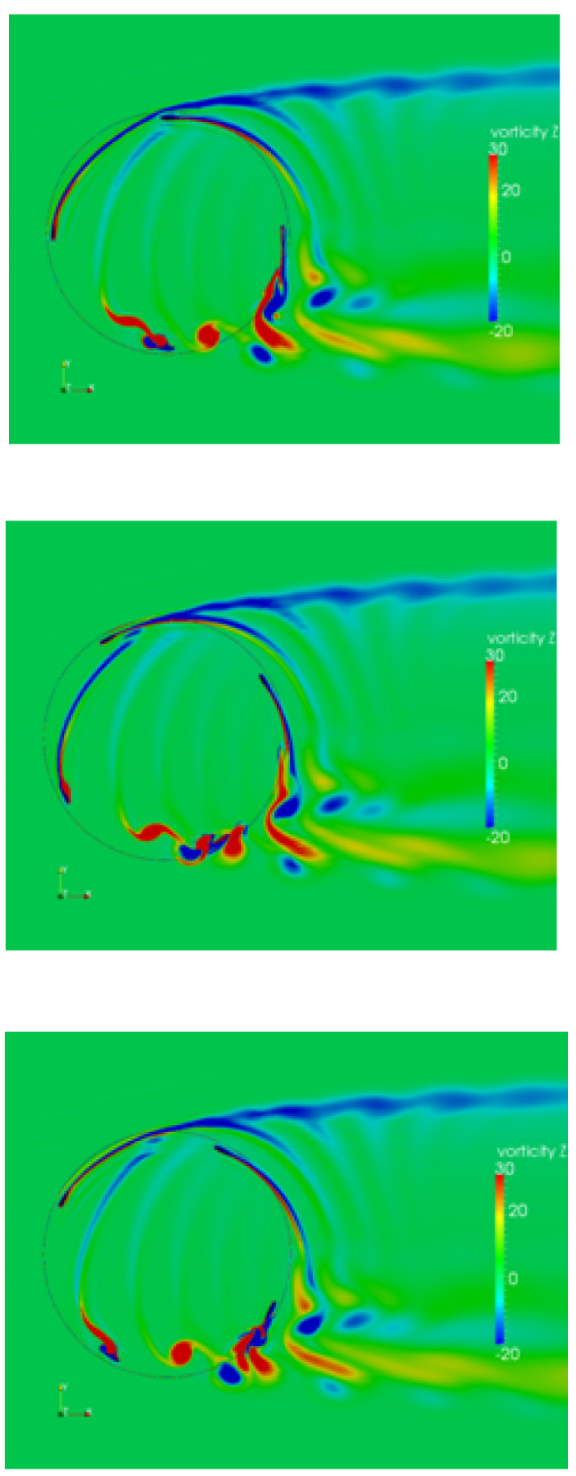

$\mathrm{TSR}=3$

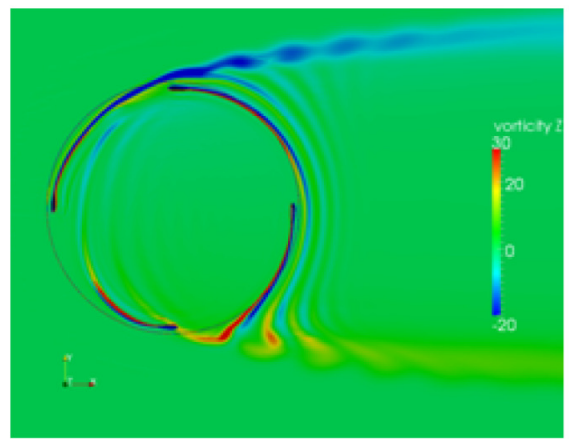

(a)

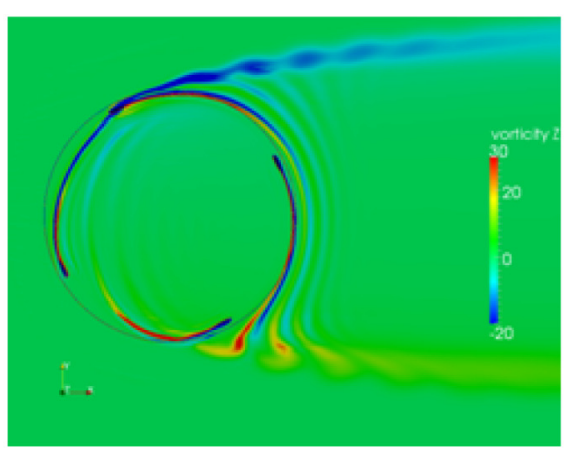

(b)

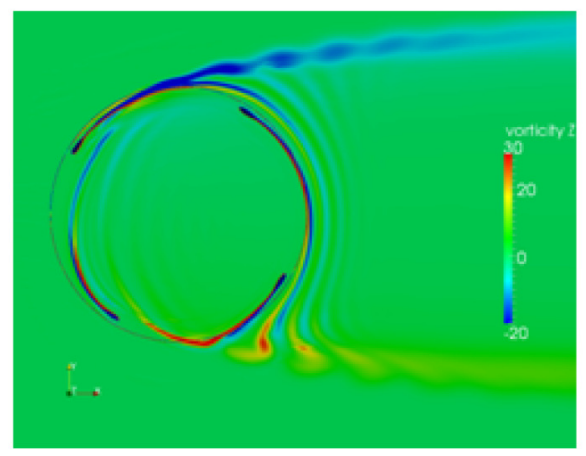

(c)

Fig. 2. Comparison of vorticity contours for TSR $=2$ (left) and TSR $=3$ (right): (a) $0^{\circ}$ position; (b) $30^{\circ}$ position and (c) $60^{\circ}$ position.

blade-vorticity interaction. The interaction is more obvious for TSR $=2$ compared to TSR $=3$ because the blades encounter a higher angle of attacks at lower TSR ratios.

Moreover, increasing the angle of attack advances the separation of flow on the suction side of the blades (the left blade in Fig. 2a). The shed vorticity from the trailing edge of the blades may collide with the other blades (Figs. 2b to 2d). It is also demonstrated that the lower the TSR, the wider extension of the wake formed behind the VAWT.

Figure 3 illustrates the $C_{p}$ results with respect to time for TSR $=3$. It should be stated that the value of $C_{p}$ demonstrates a transient behavior before reaching a periodic pattern. The averaged calculated value of $C_{p}$ is based on the last two cycles after observing a periodic behavior. 


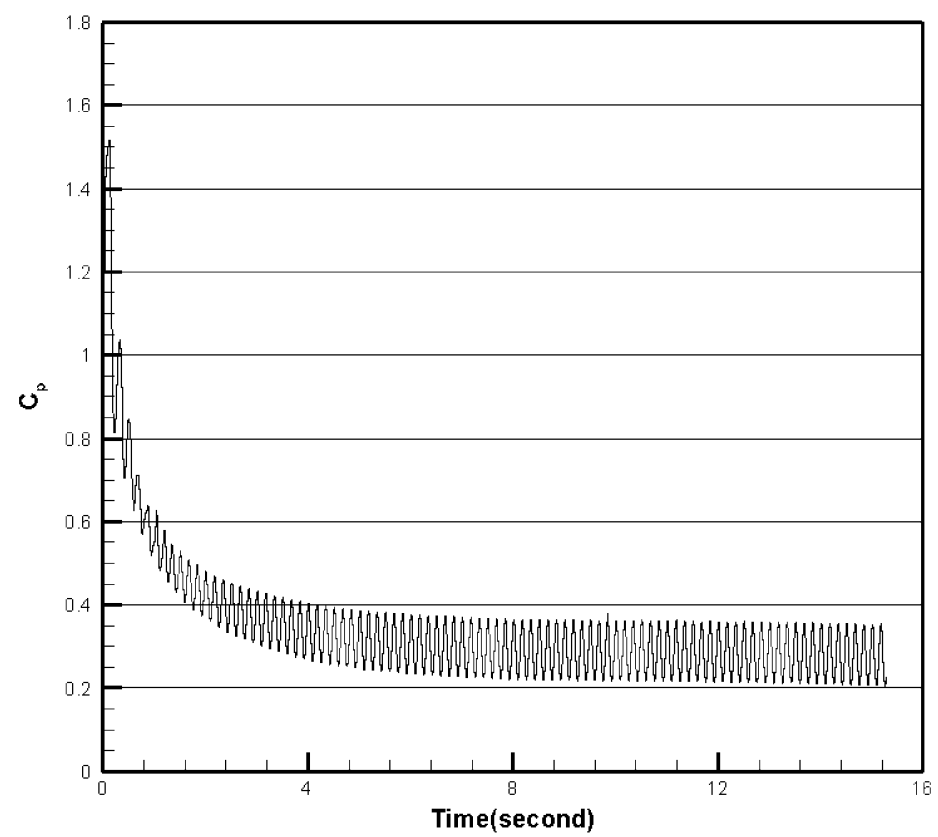

Fig. 3. $C_{p}$ versus time for TSR $=3$.

\subsection{Grid Convergence Study}

A consistent manner in reporting the results of a grid convergence was developed by Roache [14]. The Grid Convergence Index (GCI), which is based on Richardson Extrapolation (RE), is considered to be the most acceptable and recommended method employed for the discretization error estimation. In this study, grid convergence analysis has been performed among three meshes with the specifications given in Table 4 . The first step is to provide a grid or mesh size $(h)$ as follows:

$$
h=\left[\frac{1}{N} \sum_{i=1}^{N}\left(\Delta A_{i}\right)\right]^{1 / 2} .
$$

Here, $\Delta A_{i}$ is the area of the $i$ th cell, and $N$ is the number of elements used in the computation.

In this study, the coefficient of power $\left(C_{p}\right)$ is the key variable of interest and we desire to have a converged solution for this quantity. Grid refinement factor is given by

$$
r=\frac{h_{\text {coarse }}}{h_{\text {fine }}} .
$$

Based on experience, this ratio should be greater than 1.3 and the grid refinement procedure is carried out systematically. For a case where three meshes are employed, the calculation of the apparent order $p$ of the method is based on $h_{1}<h_{2}<h_{3}$ and

$$
\begin{aligned}
& r_{21}=\frac{h_{2}}{h_{1}}, \\
& r_{32}=\frac{h_{3}}{h_{2}},
\end{aligned}
$$

where $r_{21}$ and $r_{32}$ are the grid refinement factors for the first-second and second-third meshes, respectively, and $p$ is expressed by

$$
p=\frac{1}{\ln \left(r_{21}\right)}|\ln | \varepsilon_{32} / \varepsilon_{21}|+q(p)|
$$


Table 4. Discretization error for $\mathrm{TSR}=2$ and $\mathrm{TSR}=3$.

\begin{tabular}{lcc}
\hline Characteristics & \multicolumn{2}{c}{ Coefficient of Power $\left(C_{p}\right)$} \\
\cline { 2 - 3 } & TSR $=2$ & TSR $=3$ \\
\hline Fine Mesh Elements $\left(N_{1}\right)$ & $2,569,426$ & $2,569,426$ \\
Medium Mesh Elements $\left(N_{2}\right)$ & $1,429,690$ & $1,429,690$ \\
Coarse Mesh Elements $\left(N_{3}\right)$ & 798,919 & 798,919 \\
$r_{21}$ & 1.34 & 1.34 \\
$r_{32}$ & 1.34 & 1.34 \\
$\Theta_{1}$ & 0.3168 & 0.2809 \\
$\Theta_{2}$ & 0.3067 & 0.2796 \\
$\Theta_{3}$ & 0.2784 & 0.2527 \\
$p$ & 3.52 & 5.36 \\
$\Theta_{\mathrm{ext}}^{21}$ & 0.3224 & 0.2866 \\
$e_{a}^{21}$ & $3.1 \%$ & $1.9 \%$ \\
$e_{\mathrm{ext}}^{21}$ & $1.7 \%$ & $0.48 \%$ \\
$\mathrm{GCI}_{\text {fine }}^{21}$ & $2.2 \%$ & $0.64 \%$ \\
\hline
\end{tabular}

$$
\begin{gathered}
q(p)=\ln \left(\frac{r_{21}^{p}-s}{r_{32}^{p}-s}\right), \\
s=1 \cdot \operatorname{sgn}\left(\frac{\varepsilon_{32}}{\varepsilon_{21}}\right),
\end{gathered}
$$

where $\varepsilon_{32}=\Theta_{3}-\Theta_{2}, \varepsilon_{21}=\Theta_{2}-\Theta_{1}$, and $\Theta_{i}$ corresponds to the key variable in the $i$ th mesh. It should be mentioned that for $r=$ constant, $q(p)=0$. On the other hand, extrapolated values of the objective variable can be calculated using the following equation:

$$
\Theta_{\mathrm{ext}}^{21}=\frac{r_{21}^{p} \Theta_{1}-\Theta_{2}}{r_{21}^{p}-1}
$$

In order to estimate GCI for the fine mesh, the approximate and extrapolated relative errors are described as follows:

$$
\begin{gathered}
G C I_{\text {fine }}^{21}=F_{s} \frac{e_{a}^{21}}{r_{21}^{p}-1}, \\
e_{a}^{21}\left|\frac{\Theta_{1}-\Theta_{2}}{\Theta_{1}}\right|, \\
e_{\mathrm{ext}}^{21}=\left|\frac{\Theta_{\mathrm{ext}}^{21}-\Theta_{1}}{\Theta_{\mathrm{ext}}^{21}}\right| .
\end{gathered}
$$

where $F_{s}$ is the safety factor, and $F_{s}=1.25$ for comparison among three meshes [7]. The calculation procedure for three chosen meshes is shown in Table 4.

Generally, the overall accuracy of the numerical solution can be improved by refining the grids. As a result, according to Table 4, the numerical uncertainty in the fine grid GCI for Power Coefficient $\left(C_{p}\right)$ in TSR 2 and 3 are reported as 2.2 and $0.64 \%$, respectively. It is clear from this analysis that a fine mesh is needed for spatially converged results. The estimated error on the fine mesh is $1.7 \%$ for TSR 2 and $0.5 \%$ 


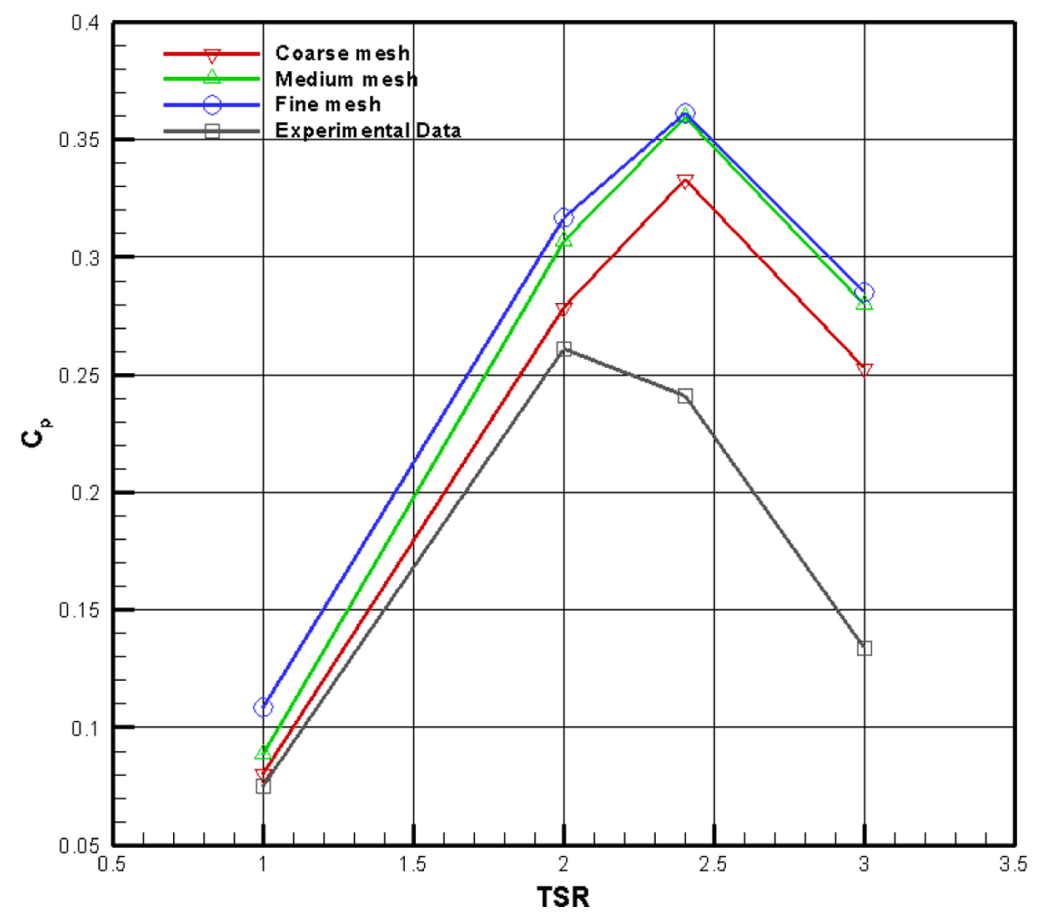

Fig. 4. Comparison of CFD and experimental results for $C_{p}$ with respect to TSR.

for TSR 3. As noted in the previous section, the TSR 2 flow has more vortices shed than the flow at TSR 3. Therefore our 2-D simulation requires at least 2 million elements. This is computationally very expensive particularly for 3-D simulations, as discussed later.

\subsection{Comparison with Experimental Data}

Figure 4 illustrates the comparison of the numerical results for three meshes with experimental data. For all three grids the maximum power coefficients are obtained at TSR $\approx 2.4$. As seen in Fig. 4, as the mesh is refined the $C_{p}$ values converge from below. Nevertheless all three numerical results overestimate the $C_{p}$ values - which is expected, as some features that decrease the $C_{p}$ are not included, such as the rotor hub and blade connections; but also 3-D features that are not captured in 2-D. These features result in reducing the power coefficient of the real turbine. In conclusion, comparison with experimental results may be misleading because a coarse mesh may give a better comparison while still being under-resolved.

Figure 5 presents the torque variations of one blade versus azimuth angle for two TSRs on the fine mesh. In this study a blade is rotated counterclockwise and the angle of attack is changed accordingly. At the time in which the chord and far-field flow are in the same direction $\left(0\right.$ and $\left.180^{\circ}\right)$, the rotor experiences the minimum torque. By increasing the azimuth angle from 0 , torque keeps increasing until it reaches its peak at $90^{\circ}$. Afterwards the torque decreases and then a second peak is observed in the backwind at $270^{\circ}$. The results of TSR $=2$ and TSR $=3$ show almost the same pattern, except the maximum pick is higher in $\mathrm{TSR}=2$.

\subsection{3-D Simulation}

\subsubsection{3-D mesh resolution study}

Herein, we calculate the 3-D mesh sizes corresponding to the 2-D mesh used in the convergence study. The numbers of 3-D elements are summarized in Table 5. To calculate the required resolution in 3-D we 


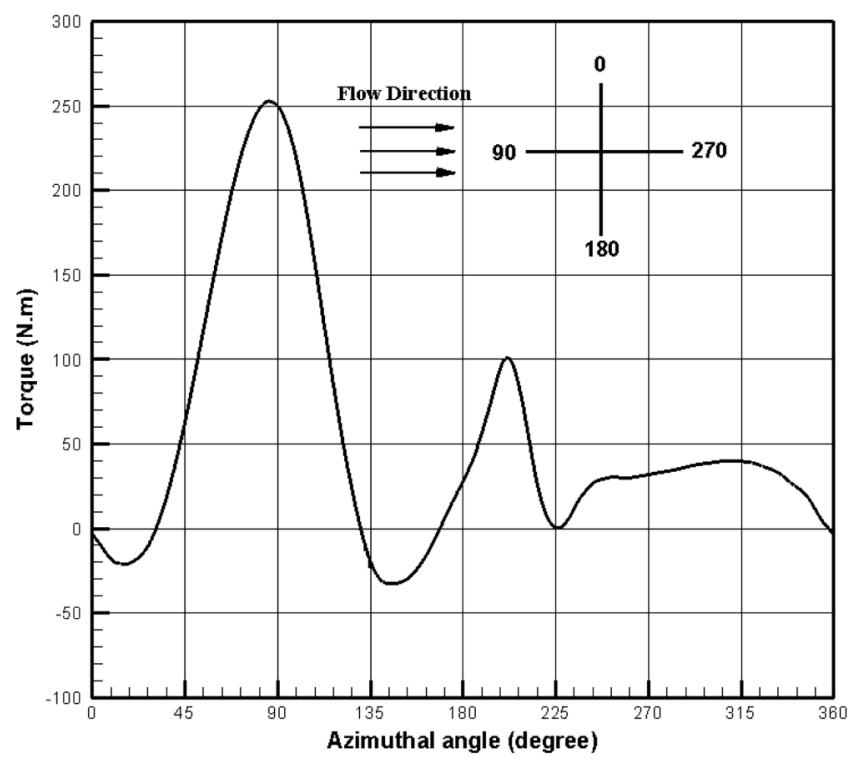

a) $\operatorname{TSR}=2$

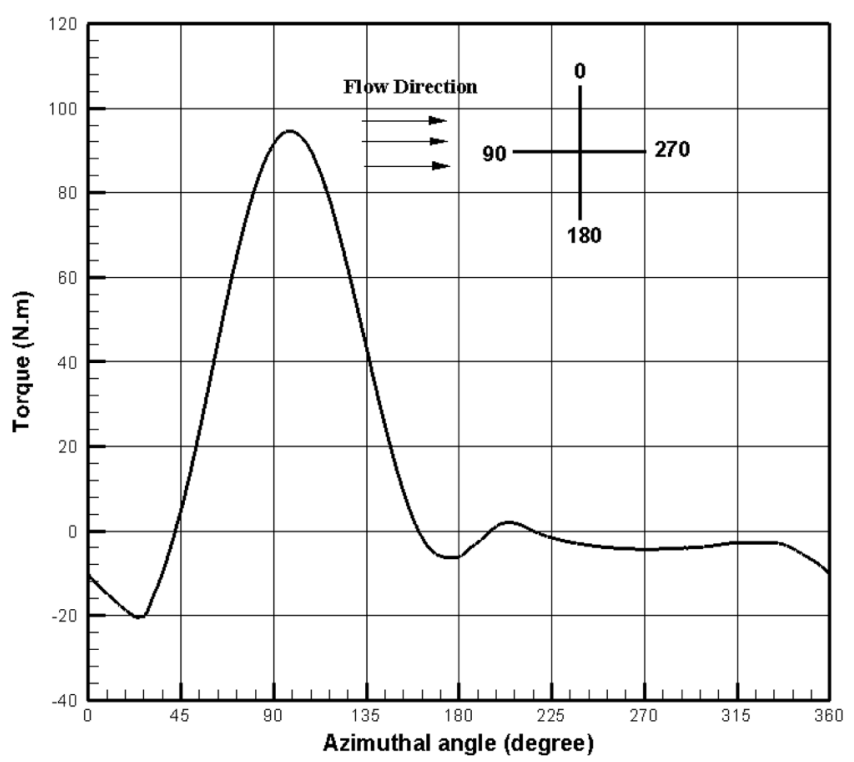

b) $\mathrm{TSR}=3$

Fig. 5. Torque variation versus azimuthal angle:(a) $\mathrm{TSR}=2$ (b) $\mathrm{TSR}=3$.

extrude, in the spanwise direction, all the 2-D meshes by 16 cells along the blade and another 16 cells between the tip of the blade and the computational boundary. The empty blade space between the tip of the blade and the computational boundary is also filled with elements. For example, the first 3-D mesh uses $444,042 \times 32=14,209,344$ elements, plus an additional $\sim 1.8$ million elements for the empty blade space. The spanwise interval size grows exponentially from the tip to the root of the blade. Using Spalart-Allmaras 
Table 5. Prediction of number of 3-D elements corresponding to 2-D mesh.

\begin{tabular}{ccc}
\hline & Number of 2-D Cells & Number of 3-D Cells (Million) \\
\hline 1 & 444,042 & 16 \\
2 & 798,919 & 27 \\
3 & $1,429,690$ & 48 \\
4 & $2,569,426$ & 87 \\
\hline
\end{tabular}

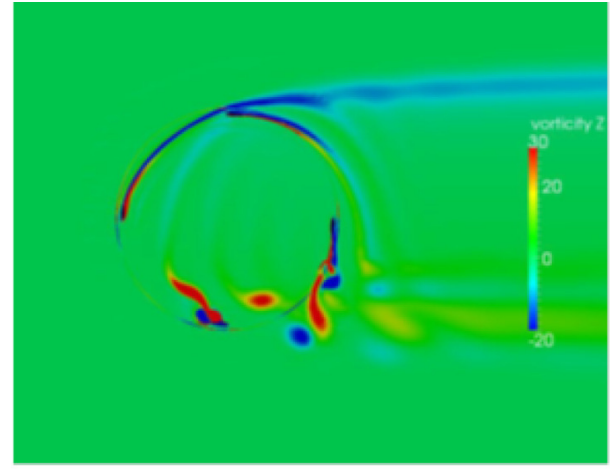

(a)

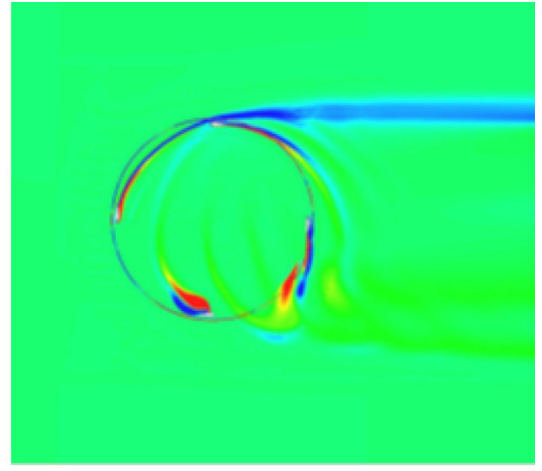

(b)

Fig. 6. Normal vorticity to the chord plane (a) 2-D simulation; (b) 3-D simulation at the blade root.

as our turbulence model, and the fact that 3-D tip vortices only disturb the flow close to the tip, justify our mesh construction. The computational time required for the first case in Table 5 is 2.5 months on a 72 processor parallel computer. This indicates that if we would like to simulate the flow around a 3-D VAWT on the finest mesh which is grid converged, it may take 14 months to calculate the $C_{p}$ for one TSR.

Although computational resources and time limitation are two main obstacles to get more accurate results for 3-D simulation at present, we still can predict an upper bound on the power coefficient from 2-D simulations. Furthermore we understand now that a finer grid will result in higher values for the power coefficient until it reaches mesh convergence. This behaviour applies both to 2D and 3D simulations.

A comparison of the normal vorticity to the chord plane in a 3-D simulation with the corresponding vorticity in a 2-D simulation at TSR $=2.0$ is depicted in Fig. 6. The 3-D simulation shows the normal vorticity contours, at the blade root, dissipating quickly. In contrast, eddies last longer and travel farther to the leeward side of the rotor with the free stream in the 2-D simulation. These are especially seen between $180^{\circ}$ and $270^{\circ}$, from the point where the blade is on the top in Fig. 6. Higher dissipation predictions in 3-D simulation may be caused by two factors: first the grid resolution is not fine enough, therefore the numerical simulation over-predicts the dissipation term. The required mesh resolution will be discussed in further detail in the next section. The second reason is related to the flow; eddies in 3-D simulation are to grow in the third directions. Therefore, the interaction of eddies changes the strength and patterns of the z-vorticity that are depicted in Fig. 6.

Figure 7 shows the $C_{p}$ variation with respect to time for the 3-D mesh at TSR $=2$. It should be mentioned that the value of $C_{p}$ follows a transient behavior before reaching a periodic convergence.

Exerted torque on each blade is reported in Fig. 8. As it is expected, the 3-D simulation results demonstrate the reduction of the turbine efficiency, compared to 2-D simulation. Figure 8 shows that the torque curve in the second half of revolution for 3-D and 2-D solutions are completely different. The main reason lies in the different prediction of separation of the flow from the blades as well as the various blade-vorticity interactions between 2-D and 3-D simulation. 


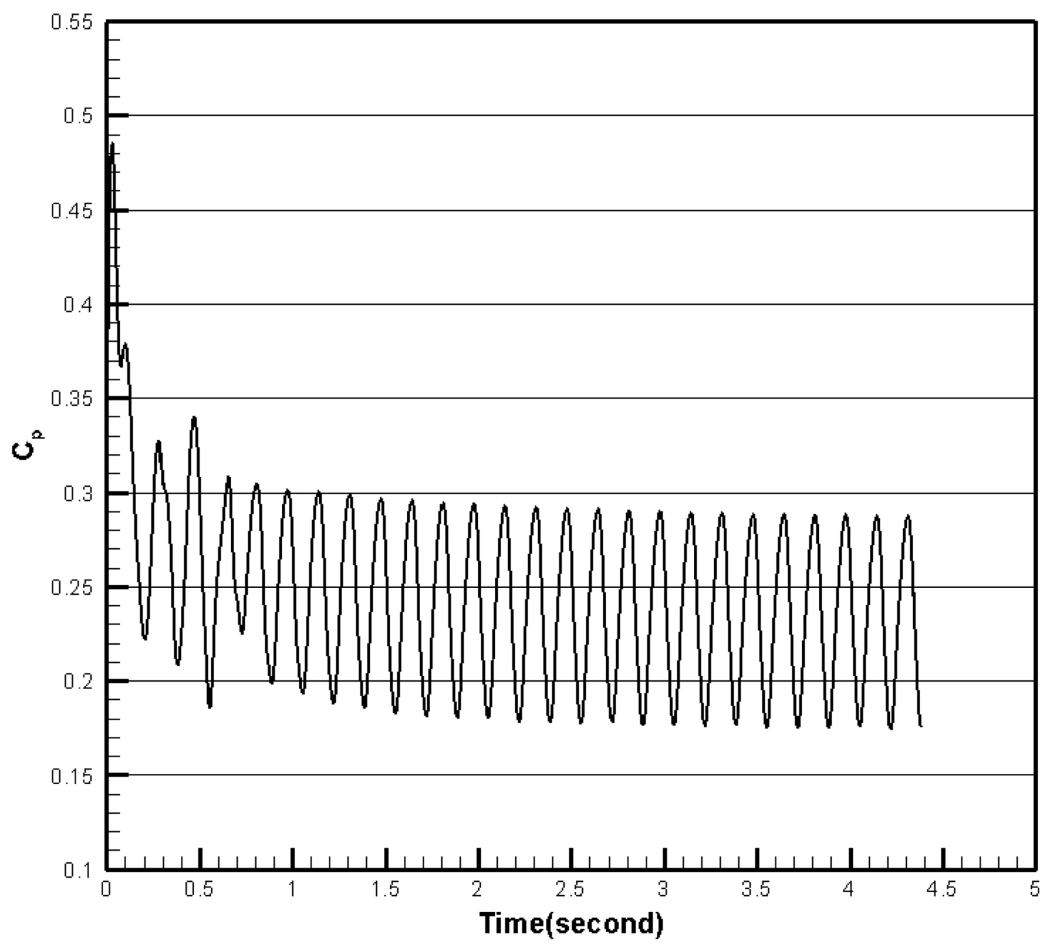

Fig. 7. $C_{p}$ variation with respect to time for the $3-\mathrm{D}$ mesh at $\mathrm{TSR}=2$.

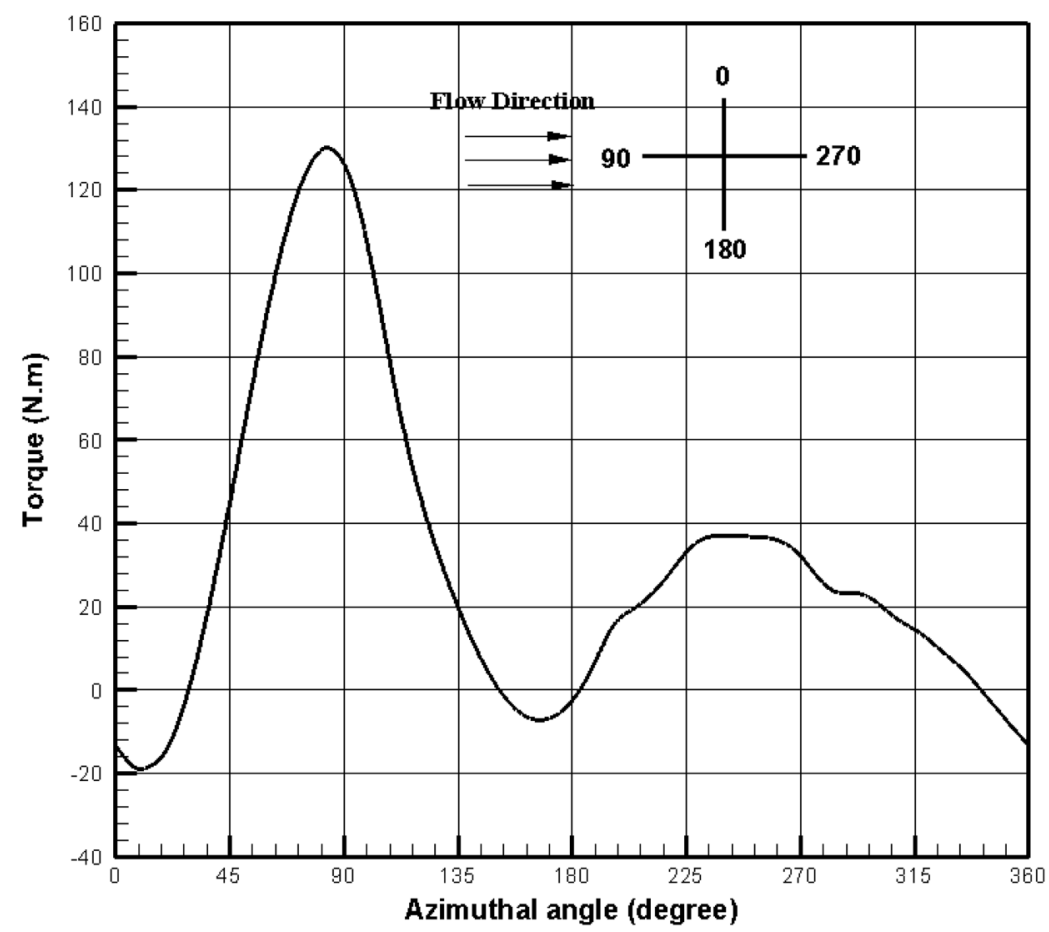

Fig. 8 . The 3-D torque variation versus azimuthal angle, $\mathrm{TSR}=2$. 


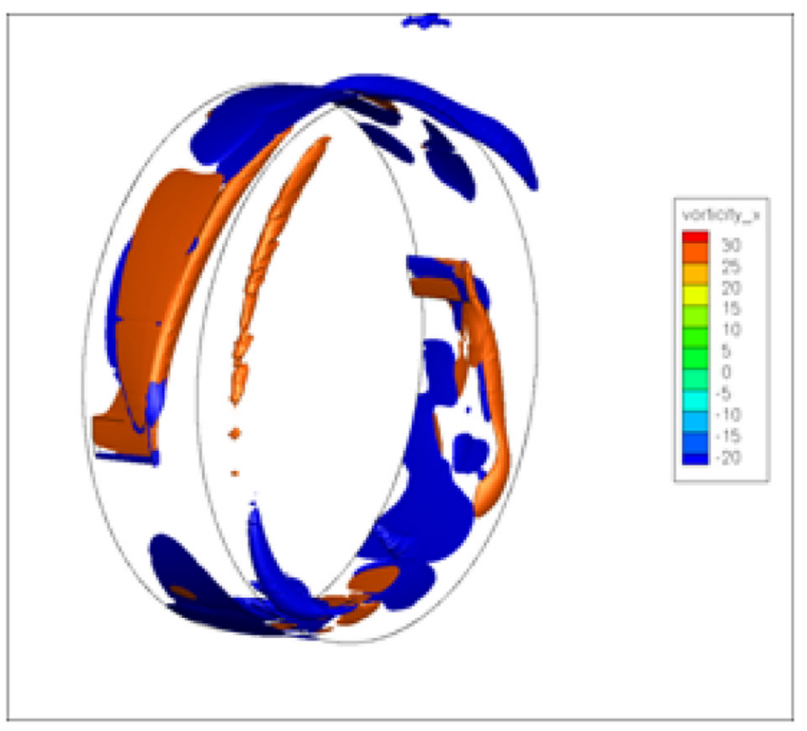

(a)

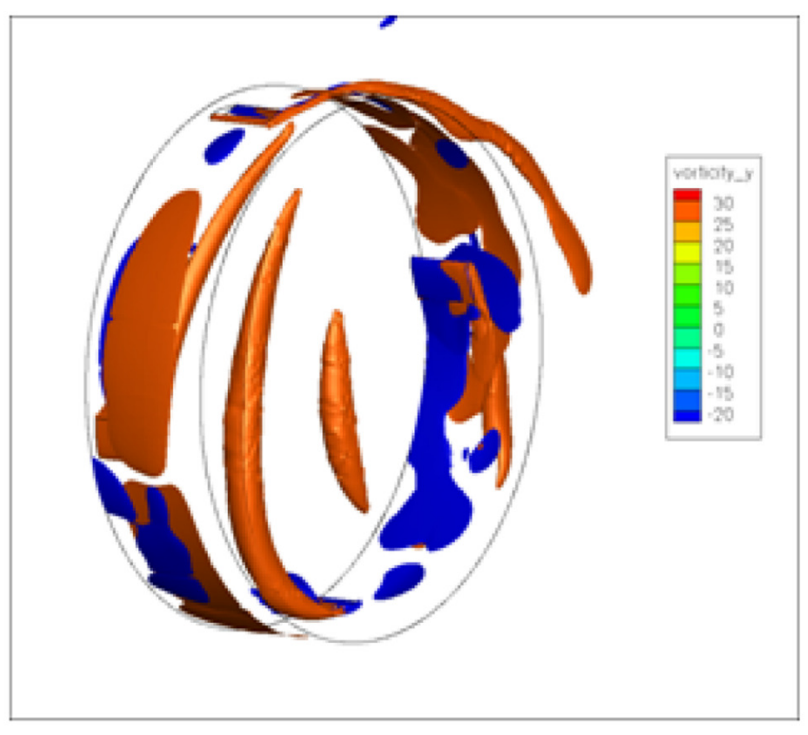

(b)

Fig. 9. (a) $X$-vorticity [1/s]; (b) $Y$-vorticity [1/s].

The average power coefficient for the 3-D simulation $\left(C_{p}=0.234\right)$ is less than the 2-D simulation predictions $\left(C_{p}=0.3168\right)$ on the fine mesh and $\left(C_{p}=0.2784\right)$ on the coarse mesh at the same TSR. The discrepancy stems from two main reasons: first, capturing the 3-D dimensional eddies and tip vortex in 3-D simulation changes the pressure distribution on the surface of the blades close to the blade tip; second, 3-D simulation is performed on a very coarse mesh which corresponds to a grid size of 444042 elements in 2-D. Comparing to the number of elements of the coarsest mesh used in Table 5 for grid convergence study, it can be concluded that the 3-D solution is not likely grid-independent. As a result we need to simulate the flow on some finer meshes that are too expensive.

\subsubsection{Analysis of tip vortex}

In this section we analyze 3-D simulation results first to capture the tip vortex. As noted, 2-D simulations are limited only to capture the vorticities that are perpendicular to the plane of simulation. These flow properties ( $z$-vorticities in our simulations) have been demonstrated and discussed in the previous section. Hence, to analyze the 3-D influence we simulate the flow on 3-D blades of VAWT at TSR $=2.0$. The averaged time step during the simulation is $\approx 0.0003 \mathrm{~s}$, then at each iteration the blades sweep $\approx 0.16^{\circ}$.

The vorticities in $x$ and $y$ directions are constructed from the 3-D results and they are shown in Fig. 9, where it is observed that both $x$ and $y$ vortices spread on and around the blades, especially concentrating close to the blade tips.

Capturing the tip vortex is a significant feature of 3-D simulations. Tip vortices are caused by a difference in pressure of the two sides of blades (pressure and suction sides). Fluid tends to flow from a higher pressure zone to a lower pressure one. Then, the natural tendency of the fluid builds vortices that travel downstream with the free stream at the tip of the blades. The phenomenon is more or less very similar to what it is seen at the tip of aircraft wings. Figure 10 illustrates the tip vortices. Note that symmetry is assumed at the base of the blades leading to identical and in phase vorticies, which is a simplification of reality.

Those are shown for the azimuth angle between $90^{\circ}$ and $180^{\circ}$. The viewer is positioned behind the trailing edge so that the tip vortex deviation from the rotation plane is observed. At the tip vortex direction is almost 


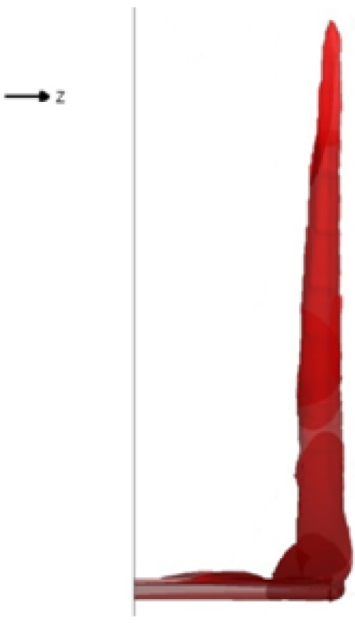

(a)

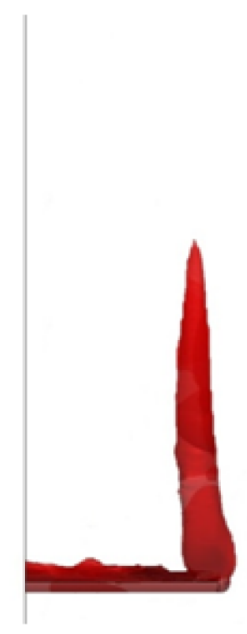

(c)

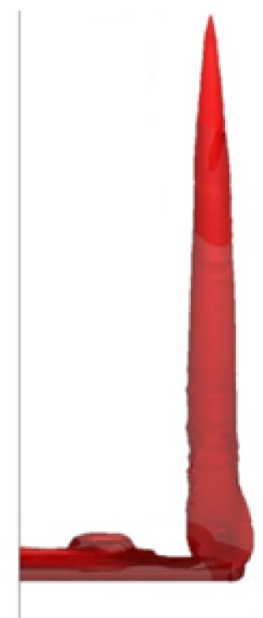

(b)

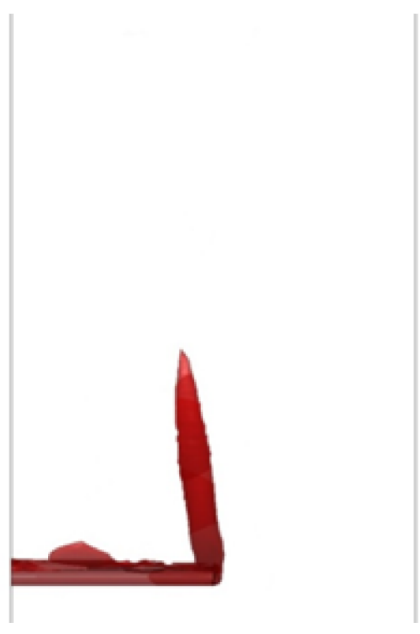

(d)

Fig. 10. Tip vortex: (a) blade at $90^{\circ}$; (b) blade at $125^{\circ}$; (c) blade at $150^{\circ}$; (d) blade at $180^{\circ}$.

the same as that of the blade tip and has not deviated left or right (Fig. 10a). When the azimuth angle increases to $125^{\circ}$ the tip vortex is shifted toward the inside of the blade (left in the figure) by $11 \%$ of the chord (Fig. 10b). The deviation grows to more than $30 \%$ of the chord at the angle of $150^{\circ}$ (Fig. 10c). Finally, the tip vortex direction makes a deviation with respect to the chord direction at $180^{\circ}$.

To understand why the tip vortices' paths deviate from the straight line, we look into the pressure contour at two sides of the blade's tip, where a blade is located at $125^{\circ}$ from top positions in Fig. 11. As is seen the pressure contours at two sides of the blade's tip differ quantitatively. It is more considerable around the blade.

Herein we compare two circled regions in the two illustrations in Fig. 11. The left image presents pressure contour at the mid-span on the blade, and the right image reports the same contour but a half-span away from the blade tip. It is observed that pressure, especially on the suction part of the blade, in the left image is significantly lower than the corresponding region in the figure on the right. We can conclude from the picture on the right that the main stream flow is dominant and the blade's influence is de- 


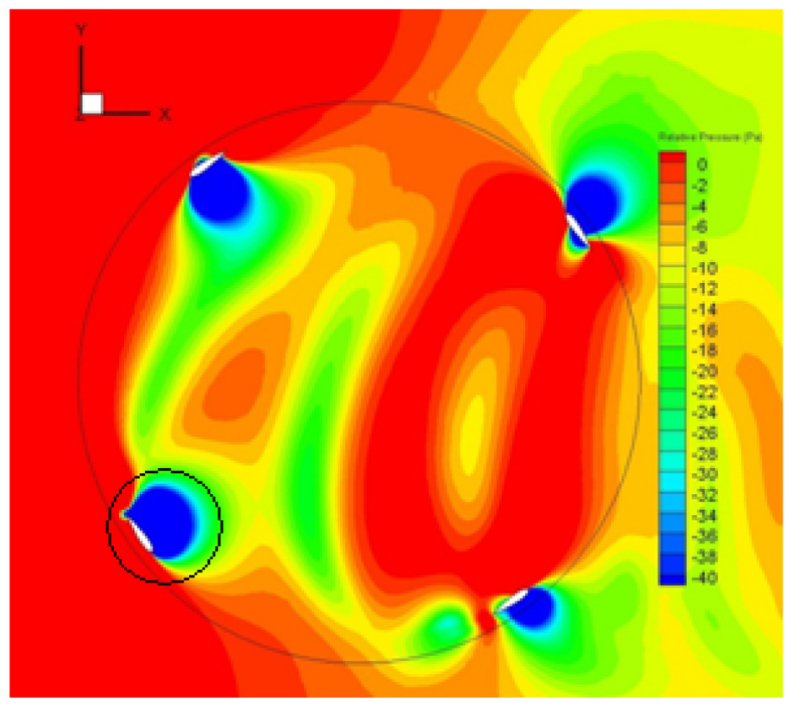

(a)

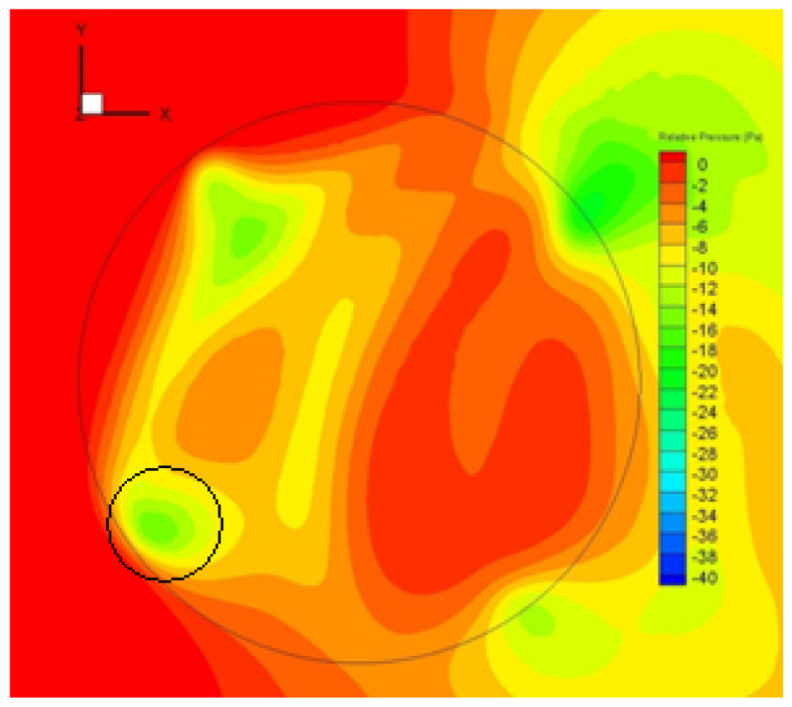

(b)

Fig. 11. Relative pressure contour: (a) the blade root; (b) mid-span away from the blade tip.

creasing with increasing spanwise distance from the blade. Therefore a higher pressure zone (Fig. 11a) pushes the tip vortices, shedding from the blades, toward a lower pressure region to the center of the blades (Fig. 11b).

\section{CONCLUSION}

A mesh convergence study based on calculating the power coefficient for 2-D flow simulations of VAWT is performed. The study shows that a fine mesh with more than 2 million elements is required. The analysis also shows that the coefficient of power converges from below; that is, the $C_{p}$ value is lower on a coarser mesh. Furthermore, the $C_{p}$ is over-estimated with 2-D simulations, as 3-D effects typically reduce the value of the $C_{p}$. We also show that 3-D simulations require a very large number of elements, on the order of 50 to 100 million, to obtain mesh convergence. We have nevertheless performed a 3-D simulation on 15 million elements to demonstrate that the tip vortex from the top of the VAWT blades moves lower as it travels downstream. In summary, computational and time limitations prevent us from performing spatially converged 3-D simulations of VAWT even with the simple S-A turbulence model; however, 2-D mesh converged results can be used appropriately as an upper value of the coefficient of power. The challenge in the next few years will be to perform accurate 3-D simulations.

\section{REFERENCES}

1. McLaren, K., Tullis, S. and Ziada, S., "CFD simulation of dynamic thrust and radial forces on a vertical axis wind turbine blade", in Proceedings of 15th Annual Conference of the CFD Society of Canada, Toronto, Canada, 2007.

2. Menter, F.R., Langtry, R.B., Likki, S.R., Suzen, Y.B., Huang, P.G. and Völker, S., "Correlation-based transition model using local variables - Part I: Model formulation”, Journal of Turbomachinery, Vol. 128, No. 3, pp. 413422, July 2006.

3. Ajedegba, J.O., Effects of blade configuration on flow distribution and power output of a Zephyr vertical axis wind turbine, University of Ontario Institute of Technology, Oshawa, Master Thesis 2008.

4. Camelli, F. and Lohner, R. L, "Combining the Baldwin Lomax and Smagorinsky turbulence models to calculate flows with separation regions", AIAA Paper No. 2002-0426, January 2002. 
5. Jiang, Z., Doi, Y. and Zhang, S. "Numerical investigation on the flow and power of small-sized multi-bladed straight Darrieus wind turbine", Journal of Zhejiang University - Science A, Vol. 8, No. 9, pp. 1414-1421, 2007.

6. Ferreira, C.J.S., Bijl, H., Van Bussel, G. and Van Kuik, G., "Simulating dynamic stall in a 2D VAWT: Modelling strategy, verification and validation with particle image velocimetry data", Journal of Physics Conference Series, Vol. 75, No. 1, pp. 012023, 2007.

7. Roache, P.J. "Perspective: A method for uniform reporting of grid refinement studies", Journal of Fluids Engineering, Vol. 116, No. 3, pp. 405-413, 1994.

8. Paciorri, R., Bonfiglioli, A., DiMascio, A. and Favini, B., "RANS simulation of a junction flow", International Journal of Computational Fluid Dynamics, Vol. 19, No. 2, pp. 179-189, 2005.

9. Biron, P.M. , Haltigin, T.W., Hardy, R.J. and Lapointe, M.F., "Assessing different methods of generating a three-dimensional numerical model mesh for a complex stream bed topography", International Journal of Computational Fluid Dynamics, Vol. 21, No. 1, pp. 37-47, 2007.

10. Hardy, R.J., Lane, S.N., Ferguson, R.I. and Parsons D.R., "Assessing the credibility of a series of computational fluid ", Hydrological Processes, Vol. 17, No. 8, pp. 1539-1560, June 2003.

11. Spalart, P.R. and Almaras, S.R ., "One-equation turbulence model for aerodynamic flows", La Recherche Aerospatiale, No. 1, pp. 5-21, 1994.

12. Open FOAM user Guide [online reference guide], OpenCFD, available from http://foam.sourceforge.net/doc/Guides-a4/UserGuide.pdf

13. Beaudoin, M. and Jasak, H., "Development of a generalized grid interface for turbomachinery simulations with OpenFOAM ", in Proceedings of Open Source CFD International Conference, Vol. 2, 2008.

14. Roache, P.J., Verification and Validation in Computational Science and Engineering, Hermosa Publishers, 1998. 